\title{
Narrative review of autoantibodies in idiopathic inflammatory myopathies
}

\author{
Yilin Liu, Yiming Zheng, Hongjun Hao, Yun Yuan \\ Department of Neurology at Peking University First Hospital, Beijing, China \\ Contributions: (I) Conception and design: All authors; (II) Administrative support: Y Yuan; (III) Provision of study materials or patients: All authors; \\ (IV) Collection and assembly of data: All authors; (V) Data analysis and interpretation: All authors; (VI) Manuscript writing: All authors; (VII) Final \\ approval of manuscript: All authors. \\ Correspondence to: Yun Yuan. Department of Neurology, Peking University First Hospital, Beijing 100034, China. Email: yuanyun2002@126.com.
}

\begin{abstract}
Objective: To discuss the characteristics of autoantigens, detection methods and roles of myositis associated autoantibodies (MAAs) and myositis specific autoantibodies (MSAs), as well as the clinical features of disease subgroups defined by MAAs/MSAs.

Background: Autoantibodies in patients with idiopathic inflammatory myopathies (IIMs) are conventionally divided into MAAs and MSAs. MAAs usually refer to autoantibodies which are also available in systematic autoimmune diseases (anti-PM/SCL, anti-Ku, anti-Ro52 and anti-U1RNP antibodies). MSAs refer to autoantibodies which were distinctive for IIM (anti-Mi-2, anti-MDA5, anti-TIF1gammma, antiNXP2, anti-SAE, anti-synthetase, anti-SRP, anti-HMGCR and anti-cN1A antibodies). The discovery and identification of novel autoantigens is a long and complicated process, which brought light in immunopathogenesis of IIMs. Detection methods of MAAs/MSAs mainly consist of monospecific methods [immunoprecipitation, enzyme-linked immune sorbent assay (ELISA) and indirect immunofluorescence] and multispecific methods [line immunoassay (LIA), dot immunoassay (DIA) and addressable laser bead assay (ALBIA)]. Patients with different MAAs/MSAs have different clinical features and require different clinical management.

Methods: The search engine PubMed (https://www.ncbi.nlm.nih.gov/pmc/) was used to research the keywords "autoantibodies", "idiopathic inflammatory myopathies", "detection methods" and "clinical features". A narrative review was conducted to literature findings from 1975 to 2020.

Conclusions: Development and validation of efficient detection methods of MAAs and MSAs help clinicians for diagnosis, classification and management of IIMs. The exploration of clinical features associated with different autoantibodies that facilitate the creation of diagnostic and classification guidelines and further clinical decision-making is of high value.
\end{abstract}

Keywords: Idiopathic inflammatory myopathies (IIMs); myositis associated autoantibodies (MAAs); myositis specific autoantibodies (MSAs); detection methods; clinical features

Submitted Jan 29, 2021. Accepted for publication Nov 26, 2021. Published online Dec 10, 2021.

doi: $10.21037 / \mathrm{atm}-21-475$

View this article at: https://dx.doi.org/10.21037/atm-21-475

\section{Introduction}

The idiopathic inflammatory myopathies (IIMs) consist of several subgroups, including overlap myositis, dermatomyositis (DM), anti-synthetase syndrome (ASS), immune-mediated necrotizing myopathy (IMNM) and inclusion body myositis (IBM). The diagnosis of IIMs requires careful assessment of clinical symptoms, elevated creatine kinase level, muscle magnetic resonance imaging (MRI) changes, electromyography findings, skeletal muscle biopsy results and analysis of myositis associated autoantibodies (MAAs) and myositis specific autoantibodies (MSAs). An integrated clinical-serologic approach for 
identifying subgroups of IIMs may be warranted (1).

MAAs are usually found in patients with overlap syndrome and less specific for IIMs. But MAAs are useful biomarker for diagnosis of IIMs and for subsequent identification of related connective tissue diseases (CTDs). MAAs mainly includes anti-PM/SCL antibodies, anti-Ku antibodies, anti-Ro52 antibodies and anti-U1RNP antibodies. MSAs are specific for IIM and each antibody correlates a distinct phenotype of IIMs with few exception (1) including DM (anti-Mi-2, anti-MDA5, antiTIF1gammma, anti-NXP2 and anti-SAE antibodies), ASS (anti-EJ, OJ, Jo1, PL7, PL12, Ha and Zo antibodies), IMNM (anti-SRP and anti-HMGCR antibodies) and IBM (anti-cN1A antibodies) $(2,3)$. Generally, one patient rarely generates two or more MSAs simultaneously (Table 1).

The discovery and knowledge of MAAs and MSAs took decades (Figure 1). Anti-Mi-2 antibodies, the first one of defined MSAs, were detected in a DM patient in 1976 (4). Since then, more than 20 kinds of autoantigens and corresponding MAAs/MSAs have been observed (Figure 2). The correlation of presence and titer of autoantibodies with clinical features has been discovered. Testing for MAAs and MSAs plays a more and more important role in diagnosis, classification and management of IIMs (5). We present the following article in accordance with the Narrative Review reporting checklist (available at https://atm.amegroups. com/article/view/10.21037/atm-21-475/rc).

\section{Methods}

The search engine PubMed (https://www.ncbi.nlm.nih.gov/ pmc/) was used to research the keywords "autoantibodies", "idiopathic inflammatory myopathies", "detection methods" and "clinical features". A narrative review was conducted to review findings of published literatures in English from 1975 to 2020 including case reports, case series, cohort studies, experiment studies and so on.

\section{Detection of MAAs/MSAs}

Multiple methods have been found to test autoantibodies (Figure 3). Immunoprecipitation, enzyme-linked immune sorbent assay (ELISA) and indirect immunofluorescence are usually considered as monospecific methods. Immunoprecipitation is widely used for detection of autoantibodies. Autoantigens from human cell lysis bind to autoantibodies from patients' serum. Then protein A/G beads binds to Fc segment of autoantibodies. Thus, antigen- antibody-protein A/G beads complex is formed. Specific antigens are extracted after centrifugation and identified by SDS-PAGE, immunoblotting or mass spectrum. Generally, immunoprecipitation is regarded as the gold-standard (6) for detecting autoantibodies because of its high sensitivity and specificity $(7,8)$. However, immunoprecipitation is not able to distinguish antigens with similar molecular weight (9). Operation of immunoprecipitation is tedious and timeconsuming and high-quality operation is needed in order to get accurate results. It is not efficient for clinical diagnostic laboratories in needing of high throughput tests.

ELISA is also widely used in diagnostic laboratories. Autoantigens are coated in a plate and bind to a specific autoantibody from patients' serum. The titer is measured by absorption photometry. It is simple to use and has high sensitivity. However, the kinds of recombinant antigens for autoantibodies testing are limited and the coating procedure can introduce loss of epitopes (10). Besides, cross-reactivity exists between some autoantibodies as a result of homologous peptide fragment, leading to decrease in specificity (11).

Indirect immunofluorescence is commonly used in antinuclear antibodies detection but is not a routine method for MSAs detection. Autoantigens existing in cultured cells or tissue slices bind to autoantibodies from patients' serum. Then fluorescent anti-human immunoglobulin reagent binds to Fc segment of autoantibodies. Each kind of autoantibodies has a specific immunofluorescent pattern which requires experienced reading $(7,8)$. Additionally, cellline based assay is rarely used for MAAs/MSAs detection. Only one credible cell-line based assay method for detection of autoantibodies against cytosolic 50-nucleotidase $1 \mathrm{~A}$ (cN1A) by using COS7 cell line was reported (12).

Line immunoassay (LIA), dot immunoassay (DIA) and addressable laser bead assay (ALBIA) are multispecific assay methods and are generally used in clinical diagnostic laboratories. These methods provide semi-quantitative information and are convenient for larger-sized samples. For LIA and DIA, the autoantigens are coated on the stripes as bands or dots. Each band or dot is coated with a different kind of autoantigen. This coating procedure can reduce the sensitivity because of loss of epitopes (13). Autoantigens bind to autoantibodies from patients' serum and antihuman immunoglobulin reagent conjugated to an enzyme bind to the Fc segment of autoantibodies. Conversion of substrates reacting with enzymes makes antigen-antibody complex visible. The color intensities of the bands or dots can be assessed by software automatically and provide 


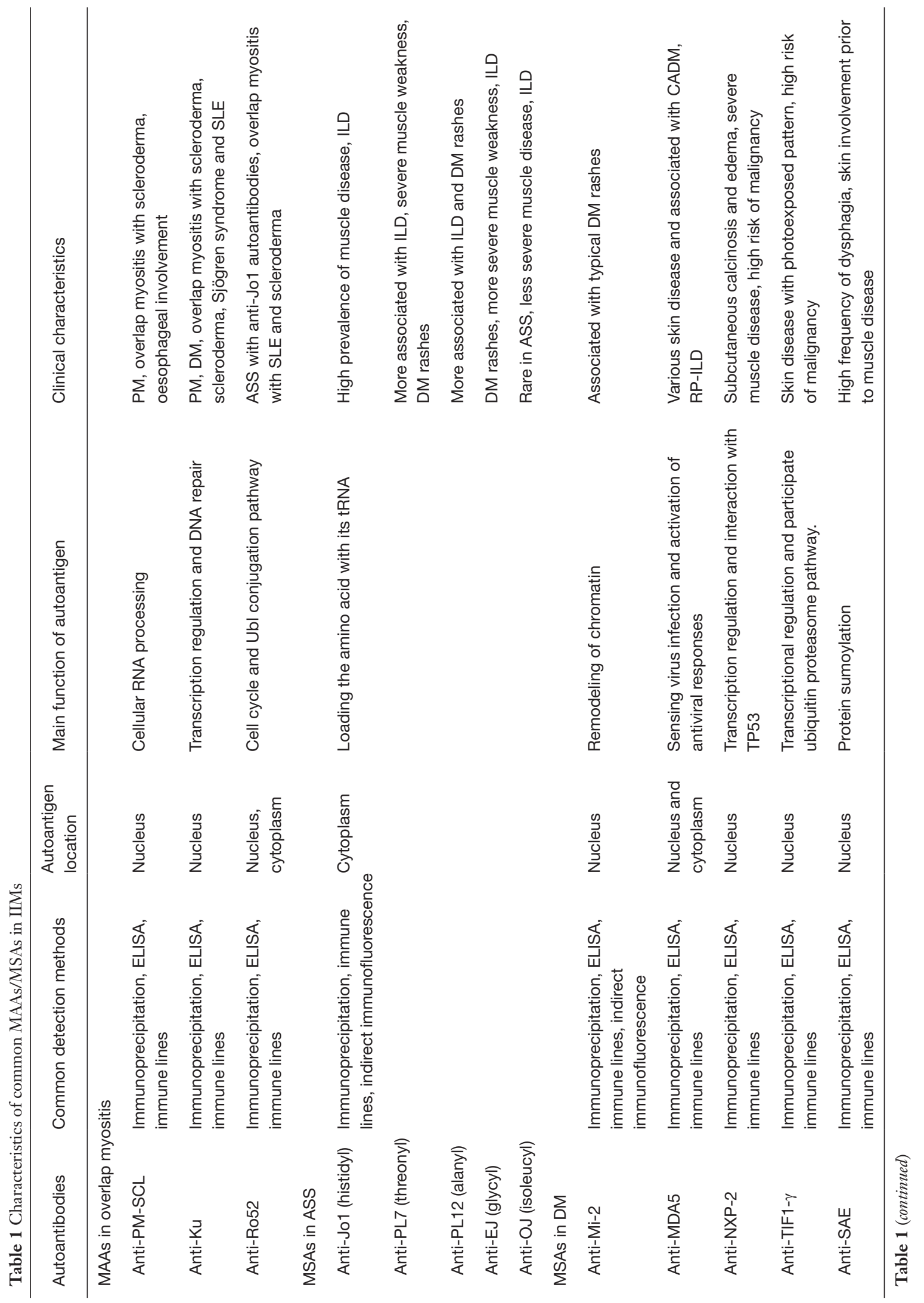




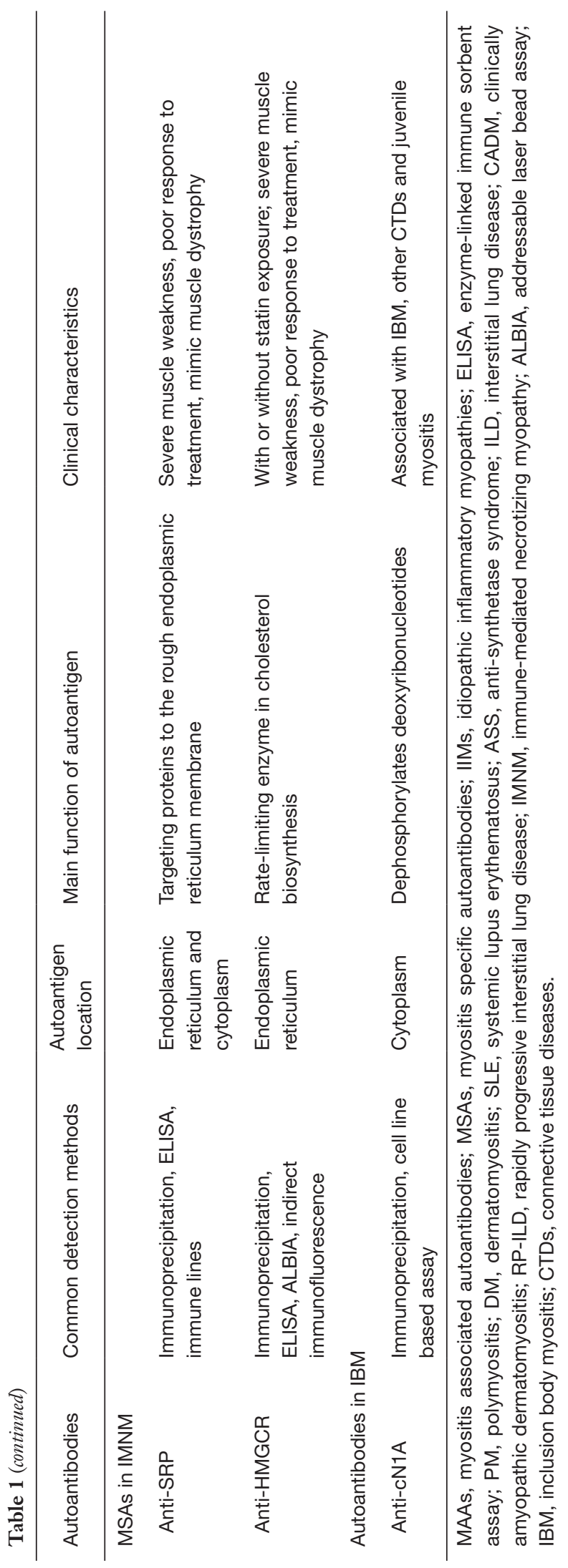

titer information. Results with low positive intensity must be interpreted prudently because it can exist in non-IIM patients with other autoimmune rheumatic diseases (13).

ALBIA is also commonly used for multispecific assay of antibodies (14). Autoantigens are coated on fluorescent beads and are incubated with patients' serum. Each bead carries a unique internal fluorescent signature and is coated with a single autoantigen. The mixture of beads are able to test multiple antibodies. The fluorescent signal intensities are assessed by flow cytometry and provide quantitative information. Fully automatic operation is available which is suitable for high-throughput screening though the testing platform and reagents can be high-cost. Commercial kits are usually made for LIA/DIA and ALBIA which are suitable for medium to large-size samples from diagnostic laboratories.

However, LIA/DIA and ELISAs are poorly applicable to detect anti-OJ autoantibodies, leading to high false negative rate. It stems from the fact that "anti-OJ antibodies" is actually "anti-OJ complex autoantibodies". Immunoprecipitation must be performed if anti-OJ syndrome is highly suspected with negative testing results by ELISA/LIA/DIA (15).

Recently, new methods such as particle-based assay are used for testing MSAs/MAAs (16) in order to strike a balance between accuracy and efficiency. The newly developed kit must be validated by immunoprecipitation and larger patient cohort is required (17).

\section{Autoantigens to MAAs/MSAs}

Autoantigens are closely associated with clinical subtypes of myositis (Table 1). Autoantigens of DM including Mi-2, NXP-2, MDA5, SAE and TIF-1 are nucleoproteins. DM patients with autoantibodies with these autoantigens share similar manifestations including typical skin lesion, interstitial lung diseases (ILDs) and muscle involvement and similar pathological changes including peripheral atrophy and obvious microangiopathy. Autoantigens of ASS are aminoacyl-tRNA synthetase in cytoplasm. Patients with ASS show similar clinical characteristics to DM but the myopathology shows necrotizing myopathy with slight microvessel damage, which makes ASS a distinct subtype of myositis. Autoantigens of IMNM including SRP and HMGCR which locate and function in endoplasmic reticulum. Patients with IMNM show severe muscle damage with necrotizing myopathy changes and show little extramuscular involvements, which makes it distinct from 


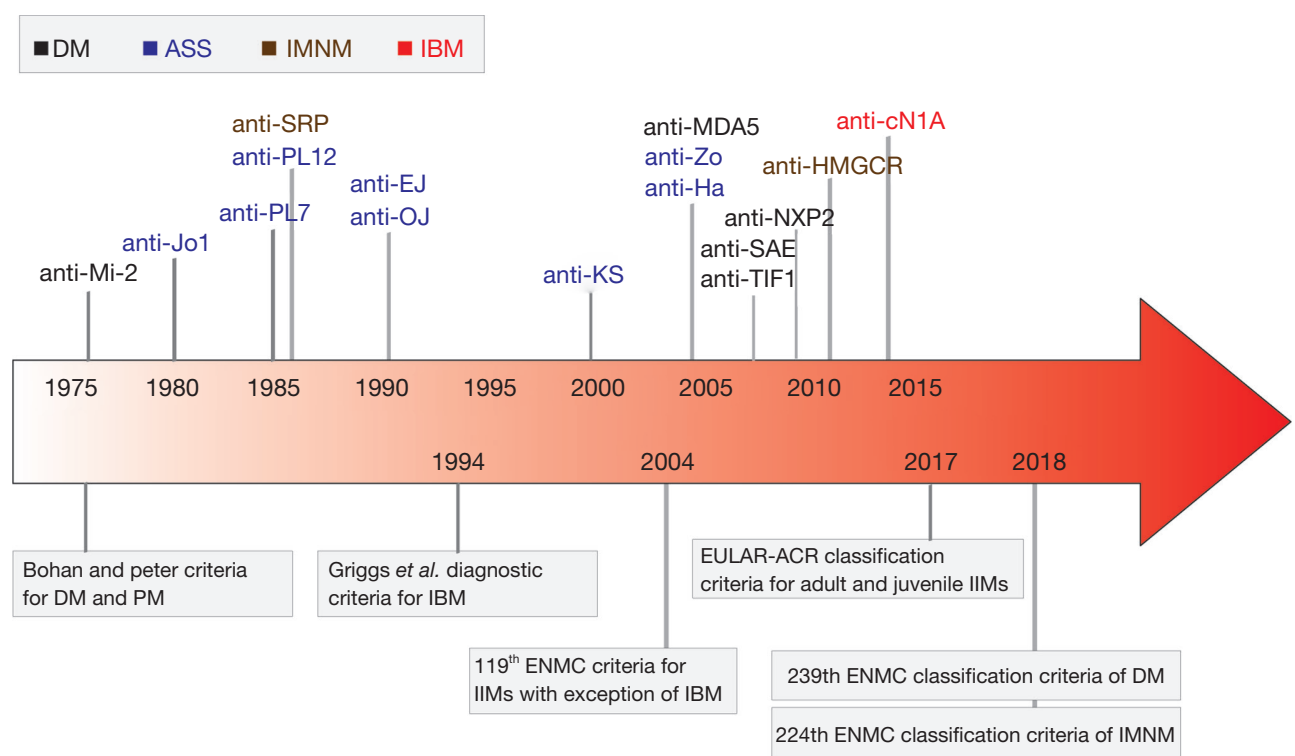

Figure 1 Timeline of discovery of MSAs and main diagnostic or classification criteria for IIMs. DM, dermatomyositis; PM, polymyositis; ASS, anti-synthetase syndrome; IMNM, immune-mediated necrotizing myopathy; IBM, inclusion body myositis; ENMC, the European Neuromuscular Centre; EULAR-ACR, the European League Against Rheumatism and the American College of Rheumatology; IIMs, idiopathic inflammatory myopathies; MSAs, myositis specific autoantibodies.

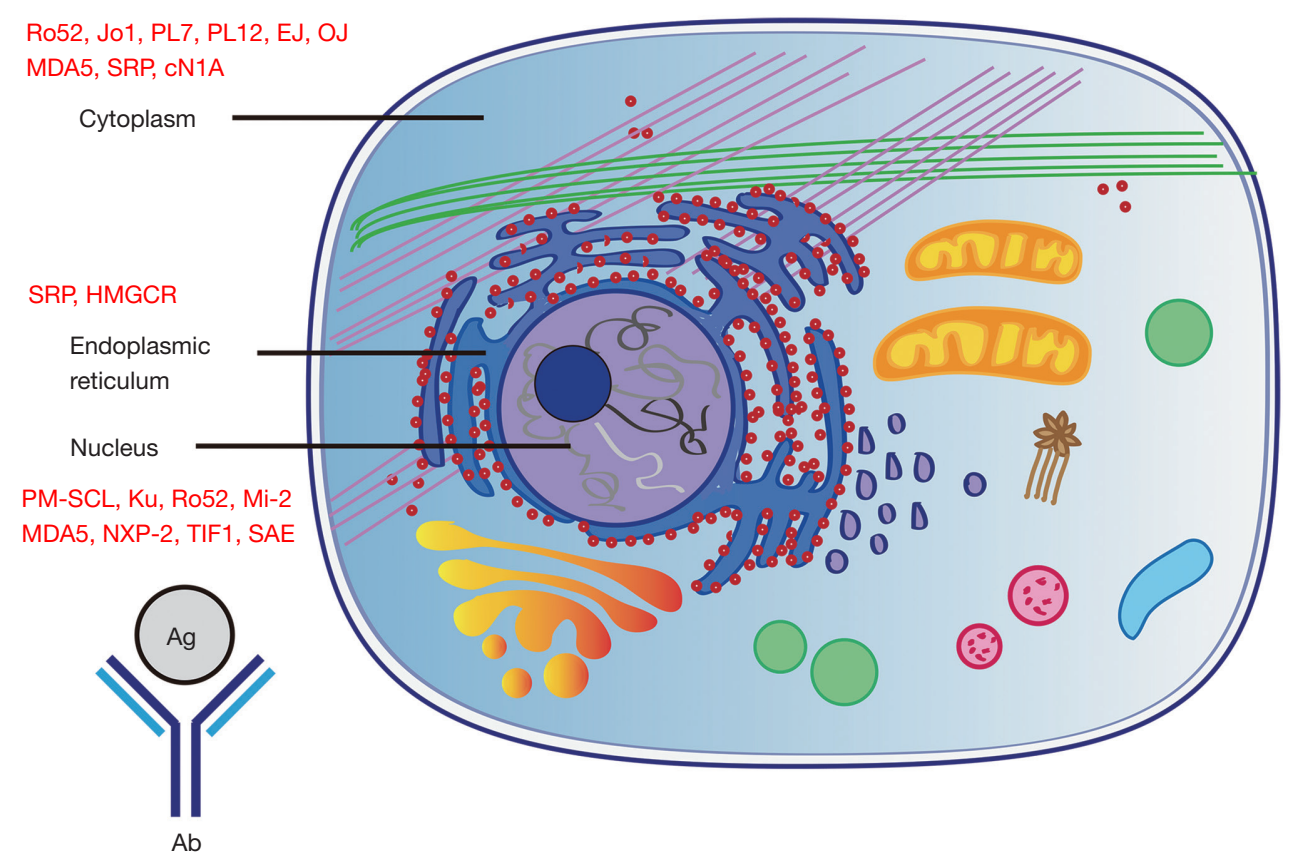

Figure 2 Distribution of autoantigens in human cells. 

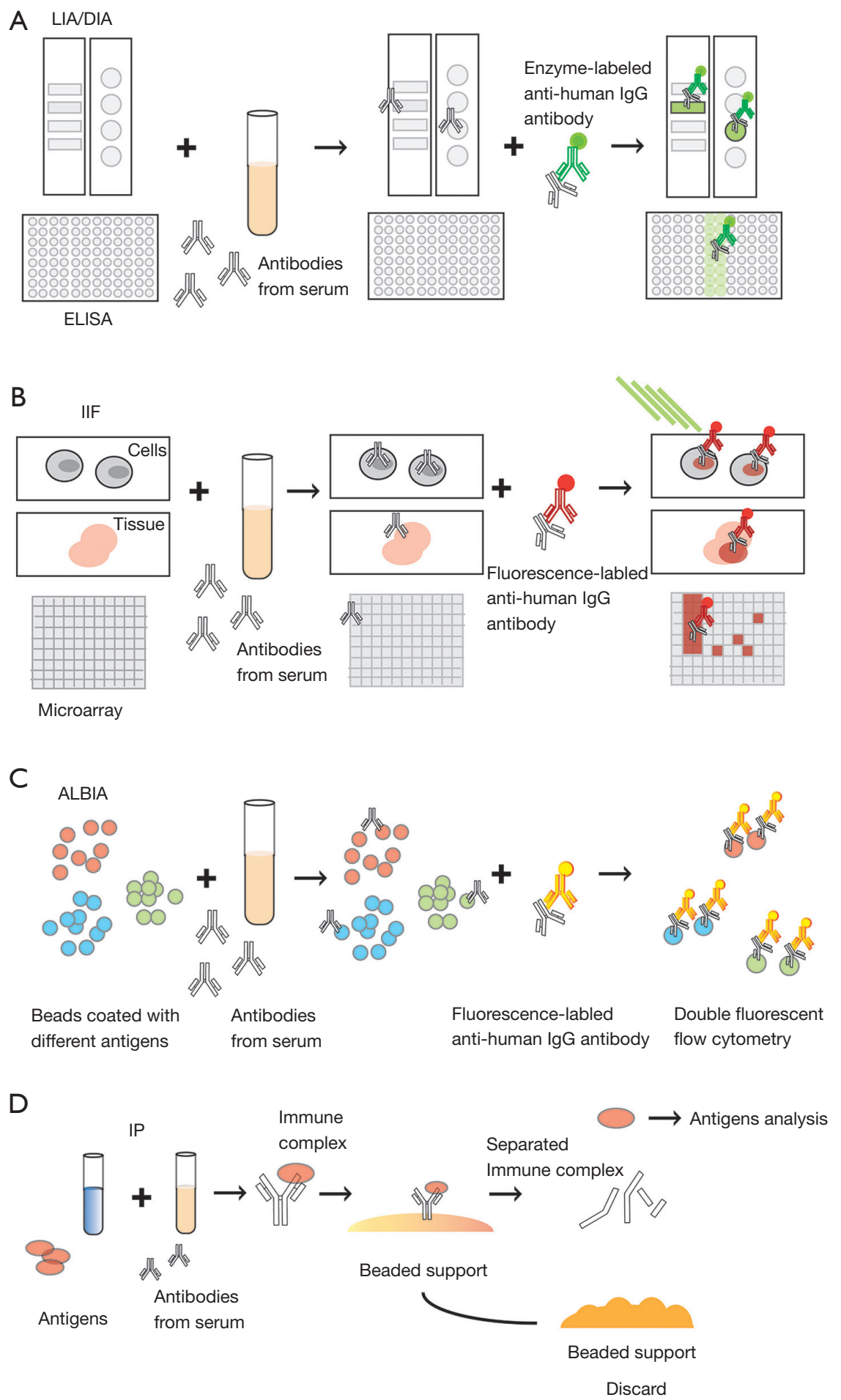

Figure 3 Mechanisms of different testing methods. (A) LIA/DIA and ELISA. (B) Indirect immune fluorescence and microarrays. (C) ALBIA. (D) Immunoprecipitation. LIA, line immunoassay; DIA, dot immunoassay; ELISA, enzyme-linked immune sorbent assay; ALBIA, addressable laser bead assay. 
ASS and DM.

In addition, the functions of each autoantigen may contribute to unique clinical features of patients with certain autoantibodies. Most autoantigens have a wide distribution and are critical to cell functions. The reason why some organs are affected in IIM while others are not is not yet clear. The autoantigens might function falsely and disturb the biological process in which they participate. For example, transcription intermediary factor 1-alpha (TIF1- $\alpha$ ) involves in the regulation of cell proliferation and apoptosis by ubiquitylation and by negatively regulating $\mathrm{p} 53$ levels (18). Transcription intermediary factor 1 -gamma (TIF $1-\gamma)$ acts as an E3 ubiquitin-protein ligase and also has a role in cell proliferation (19). Both of TIF1- $\alpha$ and TIF1- $\gamma$ involve in crucial procedures in carcinogenesis. The coexistence of anti-TIF1- $\alpha$ and anti-TIF1- $\gamma$ autoantibodies were reported to be correlated with a higher prevalence of malignancy (20).

Recently, some new autoantibodies have been shown to be associated with IIMs. Some anti-endothelial cell antibodies which bind to heat shock cognate $71 \mathrm{KD}$ protein and other chaperone proteins were identified in juvenile DM (21). The results provide new insights that anti-endothelial cell antibodies may contribute to vasculopathy of juvenile DM directly. Autoantibodies against PUF60, which locates in nucleus and plays a role in apoptosis, are associated with anti-TIF1 autoantibodies, clinically amyopathic DM $(\mathrm{CADM})$ and skin ulcerations (22). Autoantibodies against eukaryotic initiation factor 3 , a cytoplasmic complex of proteins, were found in polymyositis and showed correlation of good prognosis and response to treatment (23). Although it is increasingly difficult to find new specific autoantibodies, the discovery of new autoantibodies will bring light to studies of mechanisms of IIM.

\section{Roles of autoantibodies in myositis}

The roles of autoantibodies in the pathogenesis of myositis are not clear. There is no evidence to prove that autoantibodies associated with overlap syndrome, DM and ASS participate in the damage of muscle directly. However, the titer of autoantibodies in these disease might correlate with disease activity (24) and treatments response (25). On the contrary, anti-SRP antibodies and anti-HMGCR antibodies introduced muscle atrophy and impaired muscle regeneration (26) and participate in damage of muscle with activation of classical complement pathway (27). AntiHMGCR antibodies titers correlated with creatine kinase levels and muscle strength, indicating a possible role in pathogenesis (28).

\section{MAAs/MSAs in diagnostic criteria of IIMs}

In 2004, the 119th ENMC international workshop included MSAs detected in serum into "other laboratory criteria" for the first time while the MAAs was not mentioned (29). The criteria emphasized the significance of autoantibodies, but the associations between autoantibodies and clinical characteristics of each disease were not completely understood. Additionally, some important MSAs, such as anti-HMGCR antibodies, had not been discovered yet. In the 2017 European League Against Rheumatism/American College of Rheumatology classification criteria for adult and juvenile IIMs and their major subgroups, only antiJo1 antibodies were included due to low frequency of other antibodies documented (30). In 2017, Suzuki et al. (2) put forward an integrated diagnostic project of IIMs which proposed that diagnosis of IIMs should emphasize both muscle pathology and MSAs detection in addition to clinical features. To et al. (31) also thought that the introduction of MSAs brought better performance of myositis classification criteria because MSAs usually correlate with distinct phenotypes of IIMs. As the significance of MSAs in classification and management of IIMs has been demonstrated by multiple researches, the inclusion of MSAs by diagnostic criteria should be put on the agenda. Besides, as MAAs have been found to be associated with clinical conditions such as comorbidity and prognosis, clinical suggestions should be included in the criteria as well $(32,33)$.

\section{MAAs/MSAs in IIMs}

\section{MAAs in overlap myositis}

Overlap myositis refers to myositis combined with other types of CTDs, which might be the largest subtype within IIMs in adult patients. Anti-PM-SCL antibodies were detected in $4-12 \%$ patient with overlap syndrome in total (32) and less common in juvenile patients (34). Muscle weakness was present at onset in only $37 \%$ of anti-PMSCL positive patients while $93 \%$ finally developed weakness during follow-up (35). PM and scleroderma overlap syndrome is most commonly associated with anti-PM-SCL autoantibodies (36). We found skin changes can be various and easily ignored, which need careful examination (37). Marie et al. (38) reported that ILD was frequent (60\%) and 
oesophageal involvement occurred as a severe complication in anti-PM-SCL autoantibodies. Long-term outcome of PM/DM patients with anti-PM-SCL autoantibodies showed remission in $10 \%$ of patients and deterioration in $20 \%$ patients which suggested anti-PM-SCL autoantibodies as a poor prognostic marker. Anti-PM-SCL antibodies should be tested when patients have progressive muscle weakness along with oesophageal involvement even if MSAs tests are negative.

Anti-Ku autoantibodies was found in 1-2\% DM, 1-3\% PM, and 9-19\% overlap syndrome (32). Common clinical features of anti-Ku positive patients with IIMs included myalgia (91\%), proximal muscle weakness (89\%), and dysphagia (36\%). Rare symptoms such as camptocormia were also found in anti-Ku positive patients $(39,40)$. The anti-Ku associated overlap syndrome includes a wide range of CTDs including scleroderma, Sjögren syndrome and systemic lupus erythematosus (SLE) (41). ILD was corticosteroid-resistant (75\%) and was more frequent in patients with IIMs (82\%) than without (10.5\%) (41).

Anti-Ro/SSA antibodies, including anti-Ro60 and antiRo52 autoantibodies, are another common antibodies in overlap syndrome, which was most common in Sjogren's syndrome (42), and also found in SLE and scleroderma $(42,43)$. Anti-Ro52 autoantibodies were also common in Jo1 positive patients, who had more severe IIMs and joint involvement, symptomatic ILD and cancer and lower survival rate (44). We retrospectively categorized 1,509 suspected myositis patients and found anti-Ro52 antibodies were most common, especially accompanied by presence of antisynthetase antibodies (unpublished data).

Besides, high concentration of anti-Ro52 autoantibodies also predicted acute progressive ILD and resistance to immunosuppressant treatment in anti-Jo1 positive ASS (45). In juvenile patients with myositis, anti-Ro52 autoantibodies were associated with ILD and more severe disease (46).

Histopathological changes of overlap myositis varied and sometimes non-specific. Primary inflammation was a prominent myopathological feature $(67 \%)$ in antiPM-SCL positive IIMs patients (47). Myofiber necrosis and regeneration were found in $78 \%$ anti-Ku positive patients (48). Patterns of muscle MRI of overlap myositis with different MAAs remained unknown.

\section{MSAs in ASS}

ASS is a disease consistent of all or some clinical manifestations of ILD, Raynaud's phenomenon, mechanics hands, arthritis, fever, cutaneous rash and IIMs. A metaanalysis of 27 studies ( 3,487 patients) reported that the most common antibodies of ASS were anti-Jo1 autoantibodies (9-24\%). Other autoantibodies included PL7 (1-8\%), PL12 (1-5\%), EJ (0-3\%), OJ (0-3\%) and KS (0-3\%) (32).

Anti-EJ, anti-PL12 and anti-PL7 autoantibodies were associated with DM skin disease involvement (49). AntiJo1 autoantibodies were more associated with muscle disease while anti-PL7 and PL12 autoantibodies were more associated with ILD $(40,41)$, which were confirmed by another longitudinal cohort study (50). We found recurrent fever was common in our patients with anti-EJ antibodies and muscle damage. The mortality was thus higher in patients with anti-PL7 and PL12 autoantibodies due to presence of ILD. Another study found that patients with anti-EJ and anti-PL7 autoantibodies were more likely to present with ILD and then develop IIMs, and often developed more severe muscle weakness compared with anti-PL12, anti-OJ or anti-KS autoantibodies (49).

Myofiber necrosis or regeneration was the major histopathological change of ASS (65-75\%) $(48,51)$. Nearly half necrosis was found in perifascicular regions in patients with anti-aminoacyl-tRNA synthetases autoantibodies, especially in patients with anti-Jo1 autoantibodies (75.8\%) (52). Diffused myofiber necrosis and regeneration was occasionally observed in ASS patients with anti-OJ (10\%), anti-PL7 (4\%) and anti-Jo1 (2\%) autoantibodies (53). Endomysial fibrosis was rare (17\%) compared with other kinds of IIMs (48). Ubiquitous sarcoplasmic MHC-I expression was generally observed while MHC-II expression was mainly observed in perifascicular myofibers. C5b-9 deposition was specifically observed on sarcolemma of non-necrotic fibers in perifascicular regions rather than capillaries (54).

A cross-sectional study of thigh muscle MRI changes in ASS showed that muscle edema was pronouncedly observed in anterior groups while fatty replacement was dominantly observed in posterior groups. Anti-Jo1 positive patients showed higher muscle edema score, total edema score, total damage score and total MRI score compared with anti-Jo1 negative patients (55).

\section{MSAs in DM}

DM patients usually have skin rashes and muscle weakness, and some have ILDs, arthropathy and other rheumatic symptoms. DM is also clinically heterogeneous among and within each subgroup with different autoantibodies. Some 
subgroups had unique skin changes. Anti-SAE positive patients commonly $(75 \%)$ had distinctive diffuse darkred or pigment-like skin rashes in a Chinese cohort (56). Anti TIF1- $\gamma$ autoantibodies were associated with more extensive skin lesions which had a striking photoexposed pattern $(54 \%)$, including significantly higher frequencies of scalp rash, facial rash, V-neck rash, and back rash. Novel cutaneous features also included palmar hyperkeratotic papules, psoriasiform lesions and unique hypopigmented and telangiectatic ("red on white") patches (57). AntiMDA5 autoantibodies were found to be associated with characteristic cutaneous manifestations of hand swelling, arthritis, skin ulceration, palmar papules, mechanics hands, panniculitis, alopecia and oral ulcers (58). Subcutaneous tissue was also involved in anti-NXP-2 positive patients including subcutaneous edema (59) and subcutaneous calcinosis which occurred early and disseminated rapidly (58).

Muscle weakness always affects the proximal of limbs in DM. Patients with anti-NXP-2 autoantibodies usually had severe muscle weakness in proximal and distal of limbs, as well as in the neck. Dysphagia was common in anti-NXP-2 positive patients (60) as well as anti-SAE positive patients (61). On the contrary, anti-MDA5 autoantibodies were associated with CADM or slight muscle weakness (58). It was noteworthy that patients with anti-SAE autoantibodies commonly developed skin disease a few months prior to the onset of muscle weakness. Diagnosis of CADM should be made carefully in anti-SAE positive patients $(61,62)$.

ILD was significantly associated with anti-MDA5 autoantibodies $(58,63,64)$. Researches from both Asia $(65-67)$ and Western countries $(68,69)$ demonstrated that anti-MDA5 autoantibodies were also associated with rapidly progressive ILD, which is a predictor of poor outcome. Forty-six percent of patients died of respiratory failure within 6 months of disease onset (70).

Anti-SAE, anti-TIF1 and anti-NXP-2 autoantibodies were all associated with malignancy in IIMs $(71,72)$. Patients with anti-TIF1- $\gamma$ autoantibodies had a malignant rate of $48 \%$ (73) while had a malignant rate of $73 \%$ together with anti-TIF1- $\alpha$ autoantibodies (20). Recent studies demonstrated that anti-TIF1- $\gamma$ autoantibodies were not associated with solid tumor nor paraneoplasmic rheumatic syndrome, making it a specific marker for cancer-associated DM (74). Patients with anti-NXP-2 autoantibodies also had an increased risk of malignancy, especially in patients above 60 years old (75). The types of malignancy varied significantly and included both solid and hematological malignancies $(59,76)$.
Perifascicular atrophy and C5b-9 deposition on capillaries are characteristic histopathological changes in DM. The frequency of perifascicular atrophy varied between DM with different MSAs, including 67\% in antiMi-2 positive patients, $64 \%$ in anti-TIF1- $\gamma$ positive patients, $65 \%$ in anti-NXP-2 positive patients and $40 \%$ in antiMDA5 positive patients. Mitochondrial dysfunction was another characteristic change in DM which was common in anti-TIF1- $\gamma$ and anti-MDA5 positive patients (47). Muscle ischemic changes was common in juvenile anti-NXP-2 positive patients and indicated a severe subtype (77).

Muscle, fascial and subcutaneous edema were typical MRI changes in DM. In a study combined with muscle MRI and histopathology findings, significant association was found between inflammatory infiltrate and both muscle and fascial edema. Besides, muscle edema was found to be associated with pouched-out vacuoles (78). Differences of MRI changes between patients with different MSAs remain unknown.

\section{MSAs in IMNM}

Skeletal muscle involvement is principal in IMNM, barely accompanied with extramuscular manifestations and usually with poor recovery (79). Anti-SRP and antiHMGCR autoantibodies are crucial for the diagnosis and the prognosis $(80,81)$. Muscle atrophy and refractory to immunotherapy made it difficult to distinguish anti-SRP positive or anti-HMGCR positive IMNM from muscular dystrophy $(5,82)$.

IMNM with anti-SRP autoantibodies present generally in adults and rarely in children (83), and was associated with severe muscle weakness, a high level of creatine kinase and requirement of aggressive treatment. Younger onset was associated with more severe weakness whether patients had improved strength after treatment of immunosuppression or not. Most of them had ongoing disease activity demonstrated by elevated creatine kinase levels (82). AntiSRP autoantibody titers correlated with improvement of muscle strength after treatment, indicating it a helpful marker for monitoring of disease (84).

Although anti-HMGCR autoantibodies were firstly found in statin-associated autoimmune myopathy, not all anti-HMGCR positive patients with IMNM had a history of exposure to statins (85). In addition, anti-HMGCR autoantibodies were absent in severe self-limited statinrelated myopathy (86), strengthening the association with IMNM (81). Anti-HMGCR positive IMNM was 
occasionally observed in children and often resembled limbgirdle muscular dystrophy (81). Juvenile patients could have extremely high creatine kinase levels, severe disease and poor response to treatment $(87,88)$. Anti-HMGCR autoantibody titers were associated with creatine kinase levels and muscle strength improvement after treatment, suggesting it a marker for disease activity (89).

The main histopathological changes of anti-SRP and anti-HMGCR positive IMNM were myofiber necrosis and regeneration while extent of myofiber necrosis and regeneration was higher in anti-SRP positive patients than in anti-HMGCR positive patients. Sarcolemmal deposition of C5b-9 was also prominent in both antiSRP and anti-HMGCR positive patients. In addition, C1q and immunoglobulin $\mathrm{G}$ deposition were found close to sarcolemma. Inflammatory infiltrate was common and CD68 positive cells were most abundant (27). In chronic cases, marked endomysial fibrosis was observed which was hard to distinguish from muscle atrophy $(81,90)$.

MRI changes of IMNM include higher proportion of muscle edema, fatty replacement and atrophy while fascial and subcutaneous were less involved. Lateral rotator and gluteal muscular groups were most affected. AntiSRP positive patients had more muscle atrophy and fatty replacement than anti-HMGCR positive patients, indicating more severe muscle involvement (91). Additionally, antiSRP positive patients with striking fatty replacement were refractory to therapy, suggesting MRI a good method for monitoring treatment response (92).

\section{Autoantibodies in IBM}

Patients with IBM have finger flexor and quadriceps weakness and are easily to be misdiagnosed as chronic $\mathrm{PM}$ and receive multiple immunotherapy. Thus, the discovery of autoantibodies against cytosolic 5 '-nucleotidase 1A (cN1A or NT5C1A) brought insights into the immunopathogenic mechanisms of IBM (93). Anti-cN1A autoantibodies were detected in $30-50 \%$ of patients with IBM (94), who were more likely to be older than age 60 years at disease onset (95). Lilleker et al. (96) have reported that anti-cN1A positive patients had a higher adjusted mortality risk compared with anti-cN1A negative patients indicating it a prognostic marker. Nevertheless, anti-cN1A autoantibodies were also found in other autoimmune diseases such as Sjögren syndrome and SLE (97). Besides, Yeker et al. (98) found anti-cN1A autoantibodies were present in $27 \%$ children with juvenile myositis and juvenile idiopathic arthritis compared with $12 \%$ in healthy children. These results make it controversial whether anti-cN1A autoantibodies are specific for IBM.

Endomysial inflammatory infiltrates and rimmed vacuoles were typical histopathological changes of IBM. Increased expression LC3, TDP-43 and p62 were observed in the vacuoles. Mitochondrial deficiency in NADH staining and in cytochrome oxidase staining was also commonly observed (99). Muscle edema and fatty replacement were observed in MRI of IBM patients and the anterior group of thighs were more involved. The severity of disease in MRI increased from proximal to distal muscles (99).

\section{Conclusions}

The presence of MAAs or MSAs in patients aid clinicians to make more precise diagnosis, classification and management of IIMs. Thus, detection methods of MAAs/MSAs should be properly chosen and the results should be prudently interpreted. Further studies of roles of autoantibodies and autoantigens in immunopathogenesis will provide insights into immunotherapy and prediction of prognosis.

\section{Acknowledgments}

Funding: This work was supported by Beijing Municipal Science and Technology Commission (grant number Z191100006619034).

\section{Footnote}

Provenance and Peer Review: This article was commissioned by the Guest Editors (Hai-Feng Li and Xiangjun Chen) for the series "Laboratory Investigations in Neuroimmunological Diseases and Their Clinical Significance" published in Annals of Translational Medicine. The article has undergone external peer review.

Reporting Checklist: The authors have completed the Narrative Review reporting checklist. Available at https:// atm.amegroups.com/article/view/10.21037/atm-21-475/rc

Conflicts of Interest: All authors have completed the ICMJE uniform disclosure form (available at https://atm.amegroups. com/article/view/10.21037/atm-21-475/coif). The series "Laboratory Investigations in Neuroimmunological Diseases and Their Clinical Significance" was commissioned by the editorial office without any funding or sponsorship. 
The authors have no other conflicts of interest to declare.

Ethical Statement: The authors are accountable for all aspects of the work in ensuring that questions related to the accuracy or integrity of any part of the work are appropriately investigated and resolved.

Open Access Statement: This is an Open Access article distributed in accordance with the Creative Commons Attribution-NonCommercial-NoDerivs 4.0 International License (CC BY-NC-ND 4.0), which permits the noncommercial replication and distribution of the article with the strict proviso that no changes or edits are made and the original work is properly cited (including links to both the formal publication through the relevant DOI and the license). See: https://creativecommons.org/licenses/by-nc-nd/4.0/.

\section{References}

1. Mariampillai K, Granger B, Amelin D, et al. Development of a New Classification System for Idiopathic Inflammatory Myopathies Based on Clinical Manifestations and Myositis-Specific Autoantibodies. JAMA Neurol 2018;75:1528-37.

2. Suzuki S, Uruha A, Suzuki N, et al. Integrated Diagnosis Project for Inflammatory Myopathies: An association between autoantibodies and muscle pathology. Autoimmun Rev 2017;16:693-700.

3. Benveniste O, Stenzel W, Allenbach Y. Advances in serological diagnostics of inflammatory myopathies. Curr Opin Neurol 2016;29:662-73.

4. Reichlin M, Mattioli M. Description of a serological reaction characteristic of polymyositis. Clin Immunol Immunopathol 1976;5:12-20.

5. Kuru S, Suzuki S, Ogata K, et al. Screening of autoantibodies associated with necrotizing myopathy among undiagnosed chronic myopathy. Rinsho Shinkeigaku 2017;57:562-6.

6. Damoiseaux J, Vulsteke JB, Tseng CW, et al. Autoantibodies in idiopathic inflammatory myopathies: Clinical associations and laboratory evaluation by monoand multispecific immunoassays. Autoimmun Rev 2019;18:293-305.

7. van Dooren SH, van Venrooij WJ, Pruijn GJ. Myositisspecific autoantibodies: detection and clinical associations. Auto Immun Highlights 2011;2:5-20.

8. Satoh M, Vázquez-Del Mercado M, Chan EK. Clinical interpretation of antinuclear antibody tests in systemic rheumatic diseases. Mod Rheumatol 2009;19:219-28.

9. Kang EH, Kuwana M, Okazaki Y, et al. Comparison of radioimmunoprecipitation versus antigen-specific assays for identification of myositis-specific autoantibodies in dermatomyositis patients. Mod Rheumatol 2014;24:945-8.

10. Tansley SL, Betteridge ZE, McHugh NJ. The diagnostic utility of autoantibodies in adult and juvenile myositis. Curr Opin Rheumatol 2013;25:772-7.

11. Fujimoto M, Murakami A, Kurei S, et al. Enzyme-linked immunosorbent assays for detection of anti-transcriptional intermediary factor-1 gamma and anti-Mi-2 autoantibodies in dermatomyositis. J Dermatol Sci 2016;84:272-81.

12. Yamashita S, Tawara N. Determination of cN1A Autoantibodies by Cell-Based Immunofluorescence Cytochemistry. Methods Mol Biol 2019;1901:89-94.

13. Cavazzana I, Fredi M, Ceribelli A, et al. Testing for myositis specific autoantibodies: Comparison between line blot and immunoprecipitation assays in 57 myositis sera. $\mathrm{J}$ Immunol Methods 2016;433:1-5.

14. Drouot L, Allenbach Y, Jouen F, et al. Exploring necrotizing autoimmune myopathies with a novel immunoassay for anti-3-hydroxy-3-methyl-glutaryl-CoA reductase autoantibodies. Arthritis Res Ther 2014;16:R39.

15. Hamaguchi Y, Kuwana M, Takehara K. Comparison of anti-OJ antibody detection assays between an immunoprecipitation assay and line blot assay. Mod Rheumatol 2017;27:551-2.

16. Cavazzana I, Richards M, Bentow C, et al. Evaluation of a novel particle-based assay for detection of autoantibodies in idiopathic inflammatory myopathies. J Immunol Methods 2019;474:112661.

17. Gelpí C, Pérez E, Roldan C. Efficiency of a solidphase chemiluminescence immunoassay for detection of antinuclear and cytoplasmic autoantibodies compared with gold standard immunoprecipitation. Auto Immun Highlights 2014;5:47-54.

18. Allton K, Jain AK, Herz HM, et al. Trim24 targets endogenous $\mathrm{p} 53$ for degradation. Proc Natl Acad Sci U S A 2009;106:11612-6.

19. He W, Dorn DC, Erdjument-Bromage H, et al. Hematopoiesis controlled by distinct TIF1gamma and Smad4 branches of the TGFbeta pathway. Cell 2006;125:929-41.

20. Fujimoto M, Hamaguchi Y, Kaji K, et al. Myositisspecific anti-155/140 autoantibodies target transcription intermediary factor 1 family proteins. Arthritis Rheum 2012;64:513-22.

21. Karasawa R, Tamaki M, Sato T, et al. Multiple target 
autoantigens on endothelial cells identified in juvenile dermatomyositis using proteomics. Rheumatology (Oxford) 2018;57:671-6.

22. Zhang YM, Yang HB, Shi JL, et al. The prevalence and clinical significance of anti-PUF60 antibodies in patients with idiopathic inflammatory myopathy. Clin Rheumatol 2018;37:1573-80.

23. Betteridge Z, Chinoy H, Vencovsky J, et al. Identification of a novel autoantigen eukaryotic initiation factor 3 associated with polymyositis. Rheumatology (Oxford) 2020;59:1026-30.

24. Matsuda T, Ueda-Hayakawa I, Kambe N, et al. Four cases of anti-Mi-2 antibody-positive dermatomyositis: relationship between anti-Mi-2 antibody titre and disease severity and activity. J Eur Acad Dermatol Venereol 2018;32:e233-4.

25. Gono T, Sato S, Kawaguchi Y, et al. Anti-MDA5 antibody, ferritin and IL-18 are useful for the evaluation of response to treatment in interstitial lung disease with anti-MDA5 antibody-positive dermatomyositis. Rheumatology (Oxford) 2012;51:1563-70.

26. Arouche-Delaperche L, Allenbach Y, Amelin D, et al. Pathogenic role of anti-signal recognition protein and anti3-Hydroxy-3-methylglutaryl-CoA reductase antibodies in necrotizing myopathies: Myofiber atrophy and impairment of muscle regeneration in necrotizing autoimmune myopathies. Ann Neurol 2017;81:538-48.

27. Allenbach Y, Arouche-Delaperche L, Preusse C, et al. Necrosis in anti-SRP+ and anti-HMGCR+myopathies: Role of autoantibodies and complement. Neurology 2018;90:e507-17.

28. Allenbach Y, Drouot L, Rigolet A, et al. Anti-HMGCR autoantibodies in European patients with autoimmune necrotizing myopathies: inconstant exposure to statin. Medicine (Baltimore) 2014;93:150-7.

29. Hoogendijk JE, Amato AA, Lecky BR, et al. 119th ENMC international workshop: trial design in adult idiopathic inflammatory myopathies, with the exception of inclusion body myositis, 10-12 October 2003, Naarden, The Netherlands. Neuromuscul Disord 2004;14:337-45.

30. Bottai M, Tjärnlund A, Santoni G, et al. EULAR/ACR classification criteria for adult and juvenile idiopathic inflammatory myopathies and their major subgroups: a methodology report. RMD Open 2017;3:e000507.

31. To F, Parker MJS, Ventín-Rodríguez C, et al. Including myositis-specific autoantibodies improves performance of the idiopathic inflammatory myopathies classification criteria. Rheumatology (Oxford) 2019;58:2331-3.
32. Lega JC, Fabien N, Reynaud Q, et al. The clinical phenotype associated with myositis-specific and associated autoantibodies: a meta-analysis revisiting the so-called antisynthetase syndrome. Autoimmun Rev 2014;13:883-91.

33. McHugh NJ, Tansley SL. Autoantibodies in myositis. Nat Rev Rheumatol 2018;14:290-302.

34. Rider LG, Shah M, Mamyrova G, et al. The myositis autoantibody phenotypes of the juvenile idiopathic inflammatory myopathies. Medicine (Baltimore) 2013;92:223-43.

35. De Lorenzo R, Pinal-Fernandez I, Huang W, et al. Muscular and extramuscular clinical features of patients with anti-PM/Scl autoantibodies. Neurology 2018;90:e2068-76.

36. Muro Y, Hosono Y, Sugiura K, et al. Anti-PM/Scl antibodies are found in Japanese patients with various systemic autoimmune conditions besides myositis and scleroderma. Arthritis Res Ther 2015;17:57.

37. Liu Y, Zheng Y, Gang Q, et al. Perimysial microarteriopathy in dermatomyositis with anti-nuclear matrix protein-2 antibodies. Eur J Neurol 2020;27:514-21.

38. Marie I, Lahaxe L, Benveniste O, et al. Long-term outcome of patients with polymyositis/ dermatomyositis and antiPM-Scl antibody. Br J Dermatol 2010;162:337-44.

39. De Almeida Chaves S, Moulis G, Pugnet G, et al. Camptocormia with trigeminal involvement revealing myositis with anti-Ku antibodies. Joint Bone Spine 2019;86:111-2.

40. Yoshida T, Yoshida M, Mitsuyo K, et al. Dropped Head Syndrome and the Presence of Rimmed Vacuoles in a Muscle Biopsy in Scleroderma-polymyositis Overlap Syndrome Associated with Anti-Ku Antibody. Intern Med 2018;57:887-91.

41. Rigolet A, Musset L, Dubourg O, et al. Inflammatory myopathies with anti-Ku antibodies: a prognosis dependent on associated lung disease. Medicine (Baltimore) 2012;91:95-102.

42. Robbins A, Hentzien M, Toquet S, et al. Diagnostic Utility of Separate Anti-Ro60 and Anti-Ro52/TRIM21 Antibody Detection in Autoimmune Diseases. Front Immunol 2019;10:444.

43. Defendenti C, Atzeni F, Spina MF, et al. Clinical and laboratory aspects of Ro/SSA-52 autoantibodies. Autoimmun Rev 2011;10:150-4.

44. Marie I, Hatron PY, Dominique S, et al. Short-term and long-term outcome of anti-Jo1-positive patients with antiRo52 antibody. Semin Arthritis Rheum 2012;41:890-9. 
45. Bauhammer J, Blank N, Max R, et al. Rituximab in the Treatment of Jo1 Antibody-associated Antisynthetase Syndrome: Anti-Ro52 Positivity as a Marker for Severity and Treatment Response. J Rheumatol 2016;43:1566-74.

46. Sabbagh S, Pinal-Fernandez I, Kishi T, et al. Anti-Ro52 autoantibodies are associated with interstitial lung disease and more severe disease in patients with juvenile myositis. Ann Rheum Dis 2019;78:988-95.

47. Pinal-Fernandez I, Casciola-Rosen LA, Christopher-Stine L, et al. The Prevalence of Individual Histopathologic Features Varies according to Autoantibody Status in Muscle Biopsies from Patients with Dermatomyositis. J Rheumatol 2015;42:1448-54.

48. Suzuki S, Yonekawa T, Kuwana M, et al. Clinical and histological findings associated with autoantibodies detected by RNA immunoprecipitation in inflammatory myopathies. J Neuroimmunol 2014;274:202-8.

49. Hamaguchi Y, Fujimoto M, Matsushita T, et al. Common and distinct clinical features in adult patients with antiaminoacyl-tRNA synthetase antibodies: heterogeneity within the syndrome. PLoS One 2013;8:e60442.

50. Pinal-Fernandez I, Casal-Dominguez M, Huapaya JA, et al. A longitudinal cohort study of the anti-synthetase syndrome: increased severity of interstitial lung disease in black patients and patients with anti-PL7 and anti-PL12 autoantibodies. Rheumatology (Oxford) 2017;56:999-1007.

51. Fernandez C, Bardin N, De Paula AM, et al. Correlation of clinicoserologic and pathologic classifications of inflammatory myopathies: study of 178 cases and guidelines for diagnosis. Medicine (Baltimore) 2013;92:15-24.

52. Mescam-Mancini L, Allenbach Y, Hervier B, et al. Anti-Jo-1 antibody-positive patients show a characteristic necrotizing perifascicular myositis. Brain 2015;138:2485-92.

53. Noguchi E, Uruha A, Suzuki S, et al. Skeletal Muscle Involvement in Antisynthetase Syndrome. JAMA Neurol 2017;74:992-9.

54. Aouizerate J, De Antonio M, Bassez G, et al. Myofiber HLA-DR expression is a distinctive biomarker for antisynthetase-associated myopathy. Acta Neuropathol Commun 2014;2:154.

55. Andersson H, Kirkhus E, Garen T, et al. Comparative analyses of muscle MRI and muscular function in antisynthetase syndrome patients and matched controls: a cross-sectional study. Arthritis Res Ther 2017;19:17.

56. Ge Y, Lu X, Shu X, et al. Clinical characteristics of antiSAE antibodies in Chinese patients with dermatomyositis in comparison with different patient cohorts. Sci Rep
2017;7:188.

57. Fiorentino DF, Kuo K, Chung L, et al. Distinctive cutaneous and systemic features associated with antitranscriptional intermediary factor- $1 \gamma$ antibodies in adults with dermatomyositis. J Am Acad Dermatol 2015;72:449-55.

58. Fiorentino D, Chung L, Zwerner J, et al. The mucocutaneous and systemic phenotype of dermatomyositis patients with antibodies to MDA5 (CADM-140): a retrospective study. J Am Acad Dermatol 2011;65:25-34.

59. Albayda J, Pinal-Fernandez I, Huang W, et al. Antinuclear Matrix Protein 2 Autoantibodies and Edema, Muscle Disease, and Malignancy Risk in Dermatomyositis Patients. Arthritis Care Res (Hoboken) 2017;69:1771-6.

60. Rogers A, Chung L, Li S, et al. Cutaneous and Systemic Findings Associated With Nuclear Matrix Protein 2 Antibodies in Adult Dermatomyositis Patients. Arthritis Care Res (Hoboken) 2017;69:1909-14.

61. Betteridge ZE, Gunawardena H, Chinoy H, et al. Clinical and human leucocyte antigen class II haplotype associations of autoantibodies to small ubiquitin-like modifier enzyme, a dermatomyositis-specific autoantigen target, in UK Caucasian adult-onset myositis. Ann Rheum Dis 2009;68:1621-5.

62. Fujimoto M, Matsushita T, Hamaguchi Y, et al. Autoantibodies to small ubiquitin-like modifier activating enzymes in Japanese patients with dermatomyositis: comparison with a UK Caucasian cohort. Ann Rheum Dis 2013;72:151-3.

63. Ceribelli A, Fredi M, Taraborelli M, et al. Prevalence and clinical significance of anti-MDA5 antibodies in European patients with polymyositis/dermatomyositis. Clin Exp Rheumatol 2014;32:891-7.

64. Hall JC, Casciola-Rosen L, Samedy LA, et al. Antimelanoma differentiation-associated protein 5 -associated dermatomyositis: expanding the clinical spectrum. Arthritis Care Res (Hoboken) 2013;65:1307-15.

65. Li Y, Li Y, Wu J, et al. Predictors of Poor Outcome of Anti-MDA5-Associated Rapidly Progressive Interstitial Lung Disease in a Chinese Cohort with Dermatomyositis. J Immunol Res 2020;2020:2024869.

66. Sakamoto N, Ishimoto H, Nakashima S, et al. Clinical Features of Anti-MDA5 Antibody-positive Rapidly Progressive Interstitial Lung Disease without Signs of Dermatomyositis. Intern Med 2019;58:837-41.

67. Motegi SI, Sekiguchi A, Toki S, et al. Clinical features and poor prognostic factors of anti-melanoma differentiation- 
associated gene 5 antibody-positive dermatomyositis with rapid progressive interstitial lung disease. Eur J Dermatol 2019;29:511-7.

68. Pacot L, Pouchot J, De Prost N, et al. Interstitial Lung Disease-Complicated Anti-MDA5 Antibody in Clinically Amyopathic Dermatomyositis Patients: Report of Two Cases With Distinct Clinical Features. Front Med (Lausanne) 2020;7:77.

69. Allenbach $Y$, Uzunhan $Y$, Toquet $S$, et al. Different phenotypes in dermatomyositis associated with anti-MDA5 antibody: Study of 121 cases. Neurology 2020;95:e70-8.

70. Nakashima R, Imura Y, Kobayashi S, et al. The RIG-Ilike receptor IFIH1/MDA5 is a dermatomyositis-specific autoantigen identified by the anti-CADM-140 antibody. Rheumatology (Oxford) 2010;49:433-40.

71. Yang H, Peng Q, Yin L, et al. Identification of multiple cancer-associated myositis-specific autoantibodies in idiopathic inflammatory myopathies: a large longitudinal cohort study. Arthritis Res Ther 2017;19:259.

72. Li S, Ge Y, Yang H, et al. The spectrum and clinical significance of myositis-specific autoantibodies in Chinese patients with idiopathic inflammatory myopathies. Clin Rheumatol 2019;38:2171-9.

73. Hida A, Yamashita T, Hosono Y, et al. AntiTIF1- $\gamma$ antibody and cancer-associated myositis: A clinicohistopathologic study. Neurology 2016;87:299-308.

74. Venalis P, Selickaja S, Lundberg K, et al. Association of Anti-Transcription Intermediary Factor $1 \gamma$ Antibodies With Paraneoplastic Rheumatic Syndromes Other Than Dermatomyositis. Arthritis Care Res (Hoboken) 2018;70:648-51.

75. Fiorentino DF, Chung LS, Christopher-Stine L, et al. Most patients with cancer-associated dermatomyositis have antibodies to nuclear matrix protein NXP-2 or transcription intermediary factor $1 \gamma$. Arthritis Rheum 2013;65:2954-62.

76. Ichimura Y, Matsushita T, Hamaguchi Y, et al. AntiNXP2 autoantibodies in adult patients with idiopathic inflammatory myopathies: possible association with malignancy. Ann Rheum Dis 2012;71:710-3.

77. Aouizerate J, De Antonio M, Bader-Meunier B, et al. Muscle ischaemia associated with NXP2 autoantibodies: a severe subtype of juvenile dermatomyositis. Rheumatology (Oxford) 2018;57:873-9.

78. Milisenda JC, Collado MV, Pinal-Fernandez I, et al. Correlation between quantitative and semiquantitative magnetic resonance imaging and histopathology findings in dermatomyositis. Clin Exp Rheumatol 2019;37:633-40.
79. Allenbach Y, Benveniste O. Peculiar clinicopathological features of immune-mediated necrotizing myopathies. Curr Opin Rheumatol 2018;30:655-63.

80. Wang L, Liu L, Hao H, et al. Myopathy with antisignal recognition particle antibodies: clinical and histopathological features in Chinese patients. Neuromuscul Disord 2014;24:335-41.

81. Carvalho AAS, da Silva VG, Zanoteli E, et al. Myopathy due to HMGCR antibodies in adult mimicking muscular dystrophy associated with cancer and statin exposure narrative review of the literature - case report. Ther Clin Risk Manag 2018;14:903-7.

82. Pinal-Fernandez I, Parks C, Werner JL, et al. Longitudinal Course of Disease in a Large Cohort of Myositis Patients With Autoantibodies Recognizing the Signal Recognition Particle. Arthritis Care Res (Hoboken) 2017;69:263-70.

83. Zhao Y, Liu X, Zhang W, et al. Childhood autoimmune necrotizing myopathy with anti-signal recognition particle antibodies. Muscle Nerve 2017;56:1181-7.

84. Aggarwal R, Oddis CV, Goudeau D, et al. Anti-signal recognition particle autoantibody ELISA validation and clinical associations. Rheumatology (Oxford) 2015;54:1194-9.

85. Mammen AL, Chung T, Christopher-Stine L, et al. Autoantibodies against 3-hydroxy-3-methylglutarylcoenzyme A reductase in patients with statin-associated autoimmune myopathy. Arthritis Rheum 2011;63:713-21.

86. Floyd JS, Brody JA, Tiniakou E, et al. Absence of antiHMG-CoA reductase autoantibodies in severe self-limited statin-related myopathy. Muscle Nerve 2016;54:142-4.

87. Tansley SL, Betteridge ZE, Simou S, et al. Anti-HMGCR Autoantibodies in Juvenile Idiopathic Inflammatory Myopathies Identify a Rare but Clinically Important Subset of Patients. J Rheumatol 2017;44:488-92.

88. Jiao Y, Cai S, Lin J, et al. Statin-naïve anti-HMGCR antibody-mediated necrotizing myopathy in China. J Clin Neurosci 2018;57:13-9.

89. Ramanathan S, Langguth D, Hardy TA, et al. Clinical course and treatment of anti-HMGCR antibodyassociated necrotizing autoimmune myopathy. Neurol Neuroimmunol Neuroinflamm 2015;2:e96.

90. Ikeda K, Mori-Yoshimura M, Yamamoto T, et al. Chronic Myopathy Associated With Anti-Signal Recognition Particle Antibodies Can Be Misdiagnosed As Facioscapulohumeral Muscular Dystrophy. J Clin Neuromuscul Dis 2016;17:197-206.

91. Pinal-Fernandez I, Casal-Dominguez M, Carrino JA, et al. Thigh muscle MRI in immune-mediated necrotising 
myopathy: extensive oedema, early muscle damage and role of anti-SRP autoantibodies as a marker of severity. Ann Rheum Dis 2017;76:681-7.

92. Zheng Y, Liu L, Wang L, et al. Magnetic resonance imaging changes of thigh muscles in myopathy with antibodies to signal recognition particle. Rheumatology (Oxford) 2015;54:1017-24.

93. Pluk H, van Hoeve BJ, van Dooren SH, et al. Autoantibodies to cytosolic 5'-nucleotidase $1 \mathrm{~A}$ in inclusion body myositis. Ann Neurol 2013;73:397-407.

94. Larman HB, Salajegheh M, Nazareno R, et al. Cytosolic $5^{\prime}$-nucleotidase $1 \mathrm{~A}$ autoimmunity in sporadic inclusion body myositis. Ann Neurol 2013;73:408-18.

95. Felice KJ, Whitaker CH, Wu Q, et al. Sensitivity and clinical utility of the anti-cytosolic 5'-nucleotidase $1 \mathrm{~A}$ (cN1A) antibody test in sporadic inclusion body myositis: Report of 40 patients from a single neuromuscular center.

Cite this article as: Liu Y, Zheng Y, Hao H, Yuan Y. Narrative review of autoantibodies in idiopathic inflammatory myopathies. Ann Transl Med 2023;11(7):291. doi: 10.21037/atm-21-475
Neuromuscul Disord 2018;28:660-4.

96. Lilleker JB, Rietveld A, Pye SR, et al. Cytosolic 5 '-nucleotidase $1 \mathrm{~A}$ autoantibody profile and clinical characteristics in inclusion body myositis. Ann Rheum Dis 2017;76:862-8.

97. Herbert MK, Stammen-Vogelzangs J, Verbeek MM, et al. Disease specificity of autoantibodies to cytosolic 5 '-nucleotidase 1A in sporadic inclusion body myositis versus known autoimmune diseases. Ann Rheum Dis 2016;75:696-701.

98. Yeker RM, Pinal-Fernandez I, Kishi T, et al. AntiNT5C1A autoantibodies are associated with more severe disease in patients with juvenile myositis. Ann Rheum Dis 2018;77:714-9.

99. Lilleker JB. Advances in the early diagnosis and therapy of inclusion body myositis. Curr Opin Rheumatol 2018;30:644-9. 